\title{
Centre for Human Development, Stem Cells \& Regeneration
}

The Centre for Human Development, Stem Cells and Regeneration (CHDSCR) was founded in 2004 as a cross-disciplinary research and translational program within the Faculty of Medicine at the University of Southampton. The Centre undertakes fundamental research into early development and stem cells together with applied translational research for patient benefit. The Centre has vibrant and thriving multidisciplinary research programs that harness the translational strength of the Faculty together with an innovative Stem Cell PhD program, outstanding clinical infrastructure and enterprise to deliver on this vision.

\section{Translating pioneering developmental} \& stem cell science for patient benefit Medical advances have led to a welcome increase in life expectancy. However, with an increasing aging population, this progress presents its own new challenges: increases in age-related diseases, and associated reductions in quality of life, at considerable socio-economic cost. Stem cell science and regenerative medicine offer the opportunity to enhance our understanding of tissue development and therefore the generation of specifiable tissues. Thus there is tremendous excitement from the public and research community at the prospect of treatment of a variety of diseases and tissues lost as a consequence of aging and or trauma across the medical spectrum. In 2004, the Centre for Human Development, Stem Cells and Regeneration (CHDSCR) [20] was founded within the Faculty of Medicine. The Centre, part of the GBP£10 million Institute of Developmental Sciences, comprises over 15 multidisciplinary groups working on fundamental and clinical research encompassing human development, across the spectrum of stem cell research (embryonic, fetal, adult and induced pluripotent stem cells [iPSCs]) through to translation delivery for patient benefit applying tissue engineering and regenerative medicine strategies.
The Centre benefits from excellent research facilities, including secure facilities for the handling of human stem cells; UK Home Office-approved animal research facilities; a UK Government-licensed human tissue bank and outstanding translational capacity owing to proximity to Southampton General Hospital, Wellcome Trust Clinical Research Facility and National Institute for Health Research Biomedical Research Centre and Unit. The Centre pioneered the collection and use of human embryonic and fetal tissues for the investigation of normal and abnormal development. This unique access (which has resulted in over 1500 procedures to date) has afforded use of embryonic and fetal tissues that have informed our work on human embryonic stem cells (hESCs), human embryonic germ cells (hEGs) and human embryonic/early fetal development.

\section{Our Vision:}

- Translating pioneering developmental and stem cell science for patient benefit.

Our Mission:

- Our aim is to understand early human development and fundamental stem cell biology to inform and aid our translation
Richard OC Oreffo

Centre for Human Development, Stem Cells \& Regeneration, Human Development \& Health, Institute of Developmental Sciences, University of Southampton, Southampton, SO16 6YD, UK Tel.: +442381208502 Fax: +442381205255 roco@soton.ac.uk 
and development of cell therapies for regenerative medicine.

Our Strengths:

- Interdisciplinary research excellence in basic and translational stem cell science: we are part of the Institute of Developmental Sciences and the new GBP£48 million Institute for Life Sciences;

- Translational clinical research platforms and programs: over GBP£50 million clinical translational infrastructure, including the Wellcome Trust Clinical Research Facility and National Institute for Health Research Biomedical Research Centre and Unit;

- Integrated 4 year $\mathrm{PhD}$ program in stem cells: training the next generation of stem cell scientists;

- Public understanding of science programs: including linkage to LIFELAB [21].

\section{Current research programs \& future perspective \\ Dr Ben MacArthur: systems biology of stem cells}

Stem cell fate decisions are controlled by intrinsically complex molecular regulatory networks, involving a wide variety of protein-protein and protein-DNA interactions. Due to this complexity, it is difficult to understand molecular regulation of stem cell fate at the systems level. However, computational techniques can be used to dissect this complexity and elucidate the essential molecular mechanisms that underlie stem cell fate determination. The MacArthur group combines experimental studies with mathematical models of stem cell fate regulatory networks [1,2]. Since transcription and translation are intrinsically 'noisy' processes these models include both deterministic and stochastic mechanisms.

Prof. Tom Fleming, Dr Judith Eckert \& Dr Neil Smyth: early human development

The group focuses on how environmental influences during early development affect differentiation and long-term health. Using a mouse model, the group has pioneered studies demonstrating that a maternal low-protein diet fed exclusively during the preimplantation period induces compensatory fetal and postnatal growth at a trade-off of later disease $[3,4]$. This programming is induced by the blastocyst stage involving embryonic mTORC1-sensing of maternal nutrient status triggering proliferation and endocytosis in extraembryonic lineages to protect fetal growth. Critically, embryonic stem cells derived from such blasto- cysts memorize their origins and are utilized to model disease induction and heritability, thereby reducing animal use.

Dr Ita O'Kelly \& Prof. David Wilson: embryonic $\&$ fetal development in cardiogensis

\section{$\&$ adrenogenesis}

The groups of Dr O'Kelly and Prof. Wilson have interests in human embryonic and fetal development with a focus on cardiogenesis and adrenal gland differentiation. The O'Kelly group investigates two-pore domain potassium (K2P) channels [5] and their role in adrenal development; using a human embryonic adrenal explant culture technique, cells can be isolated that have characteristics of pluripotency and the functional expression of K2P channels. The Wilson group has identified several genes that are associated with abnormal human heart development, including $N R 2 F 2$ [6]. The role of this gene in normal development is under investigation together with targeted regulation of beta-thymosin-beta- 4 (TMSB4X).

\section{Dr Franchesca Houghton: metabolomics, hypoxia \& stem cell fate}

Research in the Houghton lab is focused on understanding molecular mechanisms that regulate a range of human stem cell populations including embryonic, cancer and renal progenitor cells with a particular interest in the hypoxic niche. The group has demonstrated that a low environmental oxygen tension is not only beneficial for maintaining a highly pluripotent population of hESCs, an effect regulated by HIF- $2 \alpha$, but is also important for regulating metabolism. Recently the group has proposed energy metabolism as a novel marker to assess hESC self-renewal [7]. The work provides critical insight into mechanisms that regulate stem cell fate pertinent to a variety of diseases.

\section{Dr Sandrine Willaime-Morawek: neural stem cell biology}

The group focuses on the role of the microenvironment on the stem cells in the developing, adult or pathological brain. Using a well-established mouse model, the group has shown that a maternal low-protein diet decreased the fetal neural stem cells, highlighting the potential effect the maternal diet has on development of the offspring brain and long-term health consequences. Using human primary glioblastoma, the group has demonstrated that A-Disintegrin and Metalloproteases promote self-renewal and inhibit migration of cancer stem cells, emphasizing A-Disintegrin and Metalloproteases as a therapeutic target for brain tumors [8]. 
Prof. Richard Oreffo: skeletal stem cells \& skeletal regeneration

The Bone and Joint Research Group focuses on skeletal biology and the mechanisms involved in skeletal stem cell (SSC) (embryonic, fetal, adult, iPSC) differentiation and skeletal tissue regeneration. The group has pioneered studies on the role of nanotopography on modulation of SSC fate [9] and on developing translational approaches to repair bone and cartilage with breakthrough publications utilizing enriched SSCs with smart scaffolds [10]. The group is also interested in the mechanisms (epigenetics) of bone development, growth and regeneration, how these differ in skeletal abnormalities and diseases and to what extent bone growth is programmed during fetal life.

\section{Dr Nicholas Evans: wound healing \& bone regeneration}

Research in the Evans' lab focuses on how the mechanical, chemical and topographical properties of the extracellular matrix affect stem cell growth, division and differentiation. His research has shown a fundamental role for matrix stiffness in the early differentiation and osteogenic differentiation of embryonic stem cells, and more recently the group has begun to explore the effect that stiffness has during skin stem cell movement and differentiation during wound healing [11,12]. The Evans lab also applies regenerative medicine to musculoskeletal reparation and is developing new methods for bone regeneration by nanoparticle targeting of SSCs.

Dr Rahul Tare: cartilage bioengineering \& SSCs Research in the Tare group focuses on SSCs, cartilage bioengineering and skeletal development. The work has been instrumental in developing strategies for isolation of SSCs, elucidating the SSC phenotype and defining conditions to manipulate SSC differentiation into principal stromal lineages. A multidisciplinary approach encompassing skeletal cells, acoustofluidics and mathematical modeling has been harnessed to bioengineer neocartilage grafts [13], with the clear aspiration that the grafts can be subsequently used in the clinic to resurface osteoarthritic cartilage. The group seeks to understand the molecular basis of skeletal diseases through improved appreciation of the role of molecular chaperones/co-chaperones in skeletal development.

Dr Claire Clarkin: angiogenesis \& development Research in Claire Clarkin's laboratory focuses on how blood vessels interact with tissues and organs during development, adulthood and disease. Specifically, how tissue derived factors, such as VEGF or TGF- $\beta$ produced by endogenous mesenchymal stromal cells
(MSCs) modulate endothelial cell behavior. Evidence supporting beneficial effects of MSCs in clinical settings is often conflicting. The group has recently described unique effects of tissue-derived MSCs upon endothelial cell function, including vascular cell apoptosis and inhibition of angiogenesis [14]. These studies highlight the broad heterogeneity within MSC populations, which could be exploited clinically.

Prof. Andrew Lotery: epithelium-derived \& corneal limbal epithelium-derived stem cells Research in this group is focused on cell therapy and disease modeling for degenerative retinal diseases $[15,16]$. Using stem cells derived from readily accessible ocular tissue or iPSCs, the group is working to generate immune-matched photoreceptors and retinal pigment epithelium (RPE) cells for cell replacement. To enhance delivery of RPE cells we have developed an innovative biostable scaffold that is being tested in vitro and in vivo. The group is also studying retinal disease modeling using patient-specific or genotype-specific iPSCs. This will provide insight into how genetic defects and environmental risk factors impact on the development and progression of retinal diseases.

\section{Training the next generation of stem cell $\&$ regenerative medicine scientists}

We are developing the next generation of stem cell and regenerative medicine scientists, able to work across boundaries and between multiple disciplines capable of translating basic discovery science into clinical research for patient benefit. We have developed a 4-year $\mathrm{PhD}$ program encompassing a year of comprehensive training in stem cells (taught modules and a variety of laboratory rotations) followed by a 3-year PhD that includes a number of transferable skills workshops and programmed events/faculty conference meetings that equip our graduates to excel in this area.

\section{Outreach}

We are particularly keen to foster an understanding and awareness of stem cell biology and regenerative medicine to the general public and have a number of educational outreach programs that harness our links to an innovative school education program, LIFELAB Southampton [21], which invites local school pupils, their parents and teachers to experience laboratorybased experiments and to learn how regenerative medicine and stem cells can impact on their lives. Our aim is to generate enthusiasm in school children and the local community and to promote public understanding of the potential contribution of stem cell research and regenerative medicine to society. 


\section{Clinical translation}

Stem cell-based cell therapy has remarkable potential for the treatment of a variety of degenerative diseases. The CHDSCR has outstanding links with the National Institute for Health Research/Wellcome Trust Clinical Research Facility, which provides a state of-the-art clinical research facility with dedicated research nursing teams, clinical trials infrastructure and, given its placement within Southampton General Hospital, a large and diverse population research base drawn from the South West and East of England. In the ophthalmology arena, the first clinical study using hESC-derived RPE for the treatment of inherited or age-related macular atrophy has recently been completed in the USA indicating cell therapy is a well-tolerated approach, with the likelihood of improvement in visual function [17]. Further clinical trials have been announced in Japan and the UK, where the safety and feasibility of using pluripotent stem cell-derived cells for the treatment of retinal degeneration will be assessed. These studies may pave the way for regenerative medicine to cure blindness. In Southampton, we are refining surgical techniques for RPE cell transplantation and deriving artificial Bruch's membranes. This work should facilitate further clinical trials in this exciting area of regenerative medicine.
In the musculoskeletal arena we have undertaken studies harnessing skeletal stem cells with graft and smart materials, including 3D-printed titanium constructs for patient treatment in areas from avascular necrosis to complex revision arthroplasty $[18,19]$. We are intending to generate living stem cell scaffold composites for application in a range of musculoskeletal conditions in the next 5 years, and to establish trials within the next 2 years.

\section{Acknowledgements}

The author would like to acknowledge and thank all members of the Centre for Human Development Stem Cells and Regeneration for their comments that have contributed to this manuscript and for continued efforts and collaborations.

\section{Financial \& competing interests disclosure}

The author is employed by the University of Southampton and holds an adjunct position at King Saud University. The author has no other relevant affiliations or financial involvement with any organization or entity with a financial interest in or financial conflict with the subject matter or materials discussed in the manuscript apart from those disclosed.

No writing assistance was utilized in the production of this manuscript.

\section{Executive summary}

- Stem cells and regenerative medicine offer the real prospect of treatment of diseases and reparation of tissues lost as a consequence of aging and or trauma.

- The Centre for Human Development, Stem Cells \& Regeneration (CHDSCR) mission is to understand early human development and fundamental stem cell biology to inform and aid our translation and development of cell therapies for regenerative medicine.

- The CHDSCR provides outstanding research facilities to enable work in early human development, across the stem cell spectrum (embryonal, adult and induced pluripotent stem cells) and outstanding translation capacity through the National Institute for Health Research/Wellcome Trust Clinical Research Facility.

- The CHDSCR is housed in the GBPf10 million Institute of Developmental Sciences with approximately $2600 \mathrm{~m}^{2}$ of accommodation and accommodates 100 researchers and support staff.

- We have developed a unique integrated 4-year PhD program in stem cells and are focused on public understanding of our programs.

\section{References}

1 MacArthur BD, Lemischka IL. Statistical mechanics of pluripotency. Cell 154(3), 484-489 (2013).

2 Méndez-Ferrer S, Michurina TV, Ferraro F et al. Mesenchymal and haematopoietic stem cells form a unique bone marrow niche. Nature 466, 829-834 (2010).

3 Eckert JJ, Porter R, Watkins AJ et al. Metabolic induction and early responses of mouse blastocyst developmental programming following maternal low protein diet affecting life-long health. PLoS ONE 7(12), e52791 (2012).

4 Sun C, Velazquez MA, Marfy-Smith $S$ et al. Mouse early extra-embryonic lineages activate compensatory endocytosis in response to poor maternal nutrition. Development 141(5), 1140-1150 (2014).
5 Mant A, Williams S, Roncoroni L, Lowry E, Johnson D and O'Kelly I. $N$-glycosylation-dependent control of functional expression of background potassium channels K2P3.1 and K2P9.1. J. Biol. Chem. 288(5), 3251-3264 (2013).

6 Al Turki S, Manickaraj AK, Mercer CL et al. Rare variants in NR2F2 cause congenital heart defects in humans. Am. J. Hum. Genet. 94(4), 574-585 (2014).

7 Forristal CE, Christensen DR, Chinnery FE et al. Environmental oxygen tension regulates the energy metabolism and self-renewal of human embryonic stem cells. PLoS ONE 8(5), e62507 (2013).

8 Bulstrode H, Jones LM, Siney EJ et al. A-Disintegrin and Metalloprotease (ADAM) 10 and 17 promote self-renewal of brain tumor sphere forming cells. Cancer Lett. 326, 79-87 (2012). 
9 Dawson JI, Kanczler JM, Yang XB, Attard GS, Oreffo RO. Clay gels for the delivery of regenerative microenvironments. Adv. Mat. 23(29), 3304-3308 (2011).

10 Dalby MJ, Gadegaard N, Oreffo RO. Harnessing nanotopography and integrin-matrix interactions to influence stem cell fate. Nat. Mater. 13(6), 558-569 (2014).

11 Evans ND, Minelli C, Gentleman E et al. Substrate stiffness affects early differentiation events in embryonic stem cells. Eur. Cell Mater. 18, 1-13 (2009).

12 Evans ND, Gentleman E. The role of material structure and mechanical properties in cell-matrix interactions. J. Mat. Chem. B. 2, 2345-2356 (2014).

13 Li S, Oreffo RO, Sengers BG, Tare RS. The effect of oxygen tension on human articular chondrocyte matrix synthesis: Integration of experimental and computational approaches. Biotechnol. Bioeng. doi:10.1002/bit.25241 (2014) (Epub ahead of print).

14 Clarkin CE, King AJ, Dhadda P et al. Activin receptor-like kinase 5 inhibition reverses impairment of endothelial cell viability by endogenous islet mesenchymal stromal cells. Stem Cells 31(3), 547-559 (2013).

15 Chen X, Thomson HA, Hossain P, Lotery AJ.

Characterisation of mouse limbal neurosphere cells: a potential cell source of functional neurons. Br. J. Ophthalmol. 96(11), 1431-1437 (2012).
Jomary C, Jones SE, Lotery AJ. Generation of light-sensitive photoreceptor phenotypes by genetic modification of human adult ocular stem cells with Crx. Invest. Ophthalmol. Vis. Sci. 51(2), 1181-1189 (2010).

17 Schwartz SD, Hubschman JP, Heilwell G et al. Embryonic stem cell trials for macular degeneration: a preliminary report. Lancet 379(9817), 713-720 (2012).

18 Dawson JI, Kanczler JM, Tare RS, Kassem M, Oreffo ROC. Bridging the gap: bone regeneration using skeletal stem cellbased strategies - where are we now? Stem Cells 32(1), 35-44 (2014).

19 Khan F, Smith JO, Kanczler JM, Tare RS, Oreffo ROC, Bradley M. Discovery and evaluation of a functional ternary polymer blend for bone repair: translation from a microarray to a clinical model. Adv. Funct. Mater. 23(22), 2850-2862 (2013).

20 The Centre for Human Development, Stem Cells and Regeneration. Www.stemcells.org.uk

21 LIFELAB. www.southampton.ac.uk/lifelab 